\title{
La inmunoterapia para ácaros con tabletas sublinguales podría reducir las exacerbaciones moderadas o severas en pacientes con asma alérgica
}

House dust mite sublingual allergen immunotherapy tablet could reduce moderate to severe allergic asthma exacerbations

\section{Objetivos}

Evaluar la eficacia y los efectos adversos de la inmunoterapia con tabletas sublinguales de ácaros del polvo (ITSL) versus placebo para reducir las exacerbaciones de asma durante períodos de reducción de corticoides inhalados $(\mathrm{Cl})$.

\section{Diseño, lugar y participantes}

Ensayo clínico aleatorizado, doble-ciego, controlado con placebo, realizado entre agosto 2011 y abril 2013 en 109 centros de 13 países europeos. Se estudiaron 834 pacientes con asma, alérgicos a ácaros, no controlados con corticoides inhalados. La eficacia fue evaluada durante los últimos seis meses del estudio cuando la dosis de $\mathrm{Cl}$ fue reducida un $50 \%$ por tres meses y retirados completamente los últimos tres meses (en los pacientes que no presentaron exacerbaciones durante los primeros tres meses).

\section{Intervención}

Los pacientes fueron aleatorizados en una relación 1:1:1 entre los que recibieron una dosis diaria de placebo $(n=277)$ o ITSL de ácaros, utilizando dos dosis diferentes de $6 \mathrm{SQ}$ (medida de activación biológica) $(n=275)$ o ITSL dosis 12 SQ $(n=282)$.

\section{Medición de resultados principales}

El resultado primario evaluado fue el tiempo trascurrido hasta la primera exacerbación de asma moderada o severa (según criterios de la American Thoracic Society y la European Respiratory Society) durante el periodo de reducción de $\mathrm{Cl}$. Los resultados secundarios fueron, disminución de síntomas de asma, aumento de anticuerpos lgG4 alérgenos específicos, cambios en los cuestionarios de calidad de vida en asma y los efectos adversos.

\section{Resultados}

El resultado primario se muestra en la tabla 1.

En cuanto a los resultados secundarios, se observó una reducción del riesgo de exacerbación con deterioro de los síntomas de asma, tanto para el grupo de dosis $6 \mathrm{SQ}$ (HR 0,72; IC95\% 0,49 a 1,02; $p=0,11$ ), como para el grupo de dosis $12 \mathrm{SQ}$ (HR 0,64; IC $95 \% 0,42$ a 0,$96 ; p=0,03$ ), con un aumento significativo de los anticuerpos IgG4 alérgeno específico.

No hubo diferencias significativas en el cuestionario de control del asma o cuestionario de calidad de vida para cualquiera de las dos dosis, ni reportes de reacciones alérgicas sistémicas graves. Los eventos adversos más frecuentes fueron leves, tales como prurito oral moderado, edema de la boca e irritación faríngea.

Tabla 1. Tiempo hasta la primera exacerbación de asma moderada o severa durante el periodo de tiempo de reducción de Corticoides inhalados.

\begin{tabular}{l|c|c|c|}
\multicolumn{1}{c|}{ Grupo } & Total pacientes (n) & $\begin{array}{c}\text { Efecto del tratamiento } \\
\text { HR (IC 95\%) }\end{array}$ & P \\
\hline Grupo Placebo & 277 & referencia & \\
\hline Grupo 6 SQ & 275 & $0,72(0,52$ a 0,99$)$ & 0,045 \\
\hline Grupo 12 SQ & 282 & $0,69(0,50$ a 0,96$)$ & 0,03 \\
\hline
\end{tabular}

${ }^{\star}$ No hubo diferencias significativas entre los dos grupos activos. HR: Hazard Ratio. IC 95\%: Intervalo de confianza del 95\%.

\section{Conclusiones}

En adultos con asma relacionada con alergia a ácaros de polvo, no bien controlada por $\mathrm{Cl}$, la adición de ITSL con ácaros a los medicamentos de mantenimiento mejoró el tiempo hasta la primera exacerbación moderada o grave del asma durante la reducción de los $\mathrm{Cl}$. La reducción se debió principalmente a un efecto sobre las exacerbaciones moderadas. Los eventos adver- sos relacionados con el tratamiento fueron frecuentes en ambas dosis activas. Se necesitan más estudios para evaluar la eficacia y la seguridad a largo plazo.

Fuente de financiamiento: ALK Dinamarca (empresa farmacéutica especializada en manejo de alergias).

\section{Comentario}

La inmunoterapia específica para alérgenos (IEA) se define como un método para administrar cantidades crecientes de alérgenos específicos a pacientes con síntomas clínicos, causados por esos alérgenos, basados en una sensibilización específica mediada por IgE, con el propósito de modular la respuesta del sistema inmune a dichos alérgenos. Los pacientes candidatos para la inmunoterapia son aquellos en los que se ha establecido un componente alérgico clínicamente importante en su enfermedad. La inmunoterapia es efectiva para el tratamiento de la rinitis alérgica, conjuntivitis alérgica, asma e hipersensibilidad a picaduras de insectos (nivel de evidencia $A)^{1}$.

IEA puede ayudar a prevenir la progresión de la enfermedad alérgica y puede ser particularmente valiosa para ayudar a los niños con rinitis alérgica y conjuntivitis a evitar el desarrollo de asma alérgica ${ }^{1}$.
La selección adecuada de los pacientes es crucial para el éxito clínico. Los desencadenantes del asma varían significativamente entre los individuos. Por lo tanto, los pacientes en quienes la exposición al alérgeno es claramente un factor importante, son más propensos a experimentar un beneficio significativo que aquellos pacientes cuyo asma es provocado en gran parte por enfermedades virales o exposición a irritantes, como el humo de tabaco.

\section{Conclusiones del comentador}

La inmunoterapia es el único tratamiento para la rinitis y el asma alérgico capaz de modificar el curso natural de la enfermedad. La correcta prescripción del tratamiento, al paciente adecuado, con la selección adecuada de alérgenos, constituye una herramienta eficiente para el manejo de estas patologías.

Carla Andrea Ritchie [ Servicio de Clínica Médica, Sección Alergia e Inmunología del Hospital Italiano de Buenos Aires. carla.ritchie@ @ospitalitaliano.org.ar ] Ritchie C. La inmunoterapia para ácaros con tabletas sublinguales podría reducir las exacerbaciones moderadas o severas en pacientes con asma alérgica. Evid Act Pract Ambul. 2017;20(1):11. Comentario de: Virchow JC, y col. Efficacy of a House Dust Mite Sublingual Allergen Immunotherapy Tablet in Adults With Allergic Asthma: A Randomized Clinical trial JAMA. 2016;315(16):1715-25.PMID: 27115376.

Referencia

1. Cox L, y col. Allergen immunotherapy: a practice parameter third update. J Allergy Clin Immunol. 2011;127(1 Suppl):S1 